FACTA UNIVERSITATIS

Series: Mechanical Engineering Vol. 15, N 3, 2017, pp. 479 - 493

https://doi.org/10.22190/FUME170911026B

Original scientific paper

\title{
DETERMINATION OF AVERAGED AXISYMMETRIC FLOW SURFACES AND MERIDIAN STREAMLINES IN THE CENTRIFUGAL PUMP USING NUMERICAL SIMULATION RESULTS
}

\author{
UDC 681.5
}

\section{Jasmina Bogdanović-Jovanović, Dragica Milenković, Živojin Stamenković, Živan Spasić}

University of Niš, Faculty of Mechanical Engineering, Serbia

\begin{abstract}
One of the most important aims in the turbo pump design is to achieve an optimal design of the pump impeller. The basic assumption in the design procedure of the impeller is that of the axisymmetric fluid flow. It can be confirmed or disputed by using the method presented in the paper, which uses the results of numerical simulation of fluid flow in the pump impeller. The method is actually a procedure for determining averaged axisymmetric flow surfaces and meridian streamlines. Furthermore, according to the obtained streamlines, a correction of the impeller blade geometry can be made (if the streamlines deviate significantly from the assumed axisymmetric ones). It is also possible to calculate the specific works of the elementary stages and compare them with the previous assumptions. The pump impeller torque can be calculated as well.
\end{abstract}

Key Words: Centrifugal Pump, Averaged Flow Surface, Meridian Streamlines, Numerical Simulations

\section{INTRODUCTION}

In view of the vast number of applications of centrifugal pumps in various systems, such as water supply, irrigation, drainage, water cooling systems, etc., it is crucial to ensure they are working in the most effective way possible. Turbo machine designing has always been a complex task, which is largely based on the designer's experience, due to many assumptions made in the process of calculating the impeller geometry. Thus, the impeller calculation has to be followed by the prototype(s) testing and later, if needed,

Received September 11, 2017 / Accepted November 27, 2017

Corresponding author: Jasmina Bogdanović-Jovanović

University of Nis, Faculty of Mechanical Engineering, A. Medvedeva 14, 18000 Niš, Serbia

E-mail: bminja@masfak.ni.ac.r 
impeller blade correction (which is usually necessary for obtaining a more efficient impeller and higher pump efficiency).

A special attention in the pump designing must be paid to the pump impeller. The basic assumption is that the fluid flow in the pump impeller is axisymmetric, which is the case of profile cascades with an infinite number of infinitely thin blades [1-4]. The continuity of the flow must be maintained, and the fluid flow energy must be increased by the required design value $[5,6]$. By averaging the flow velocities according to the circular coordinate, the fluid flow in the real pump impeller can be derived into that of the fictive impeller with an infinite number of infinitely thin blades, which creates the equal flow declination as the real pump impeller [7, 8].

An accurate determination of meridian streamlines in the centrifugal pump impeller is very important since they represent meridian traces of the axisymmetric flow surfaces in the pump impeller.

On the other hand, numerical simulations of the flow in the radial pump impeller provide for obtaining all flow parameters in the flow domain (pump impeller). There are many attempts to use the CFD results of flow in turbo pump impellers in the process of developing their optimal design [9-12].

Values of the numerically obtained flow parameters, especially of all the velocity components could be very useful, as will be presented in the method for determining averaged axisymmetric flow surfaces. This method for averaging flow parameters and calculating averaged flow surfaces according to the circular coordinate, in order to obtain a model of the fictive impeller with an infinite number of infinitely thin blades, is presented in the paper. Meridian streamlines are calculated using the integral continuity equation for the previously averaged flow parameters [8]. The comparison of the obtained meridian streamlines and the streamlines defined in the process of blade designing can be made.

Furthermore, the specific works of elementary stages in the meridian cross-section can be calculated and compared with the predefined specific works of elementary stages.

The pump impeller torque can be calculated as well, and also compared with the numerical simulation results.

\section{EQUATIONS FOR AVERAgING Flow PARAMETERS OVER CIRCUlar COORDINATE AND FLOW EQUATIONS}

Observing the universal curvilinear orthogonal coordinate system $\left(q_{1}, q_{2}, q_{3}\right)$, there are following coordinate surfaces [7,8]: meridian planes $q_{3}=$ const., axisymmetric surfaces $q_{2}=$ const. and axisymmetric flow surface $q_{1}=$ const. perpendicular to $q_{3}=$ const. (Fig. 1).

Lame's coefficients are also defined as a function of curvilinear coordinates, $L_{1}\left(q_{1}, q_{2}\right)$, $L_{2}\left(q_{1}, q_{2}\right)$ and $L_{3}=r / r_{o}$, where $r=r\left(q_{1}, q_{2}\right)$ is a radial distance from the origin of cylindrical coordinate system $(r, \varphi, z)[7,8]$.

Direction of circumferential coordinate $q_{3}\left(\vec{e}_{3}^{o}\right)$ does not have to be the same as that of the impeller rotation $(\vec{\omega})$; also, the right (positive) coordinate system is used (dashed blade lines in Fig. 1 correspond to the left (negative) coordinate system).

Using cylindrical coordinate system $(r, \varphi, z): q_{1}=z, q_{2}=r$ and $q_{3}=r_{o} \varphi\left(L_{1}=1, L_{2}=1\right.$, $L_{3}=r / r_{o}$ ), where $r_{o}$ is the radial distance in the pump inlet. 


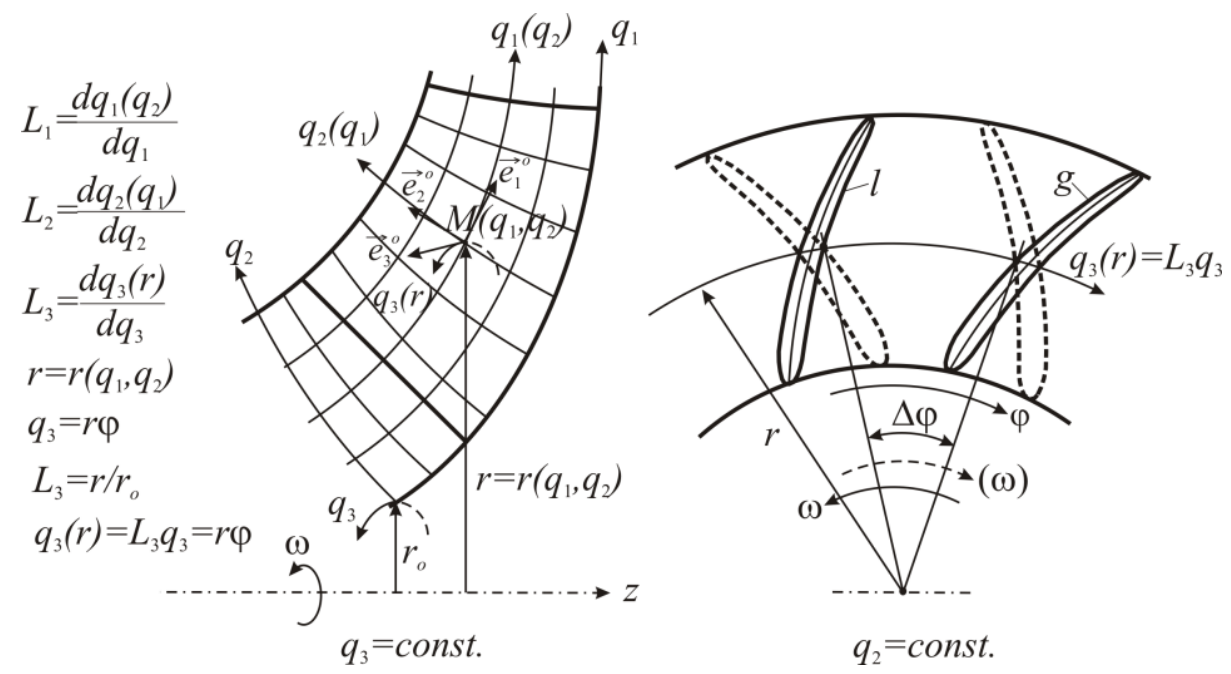

Fig. 1 Cross-section of the centrifugal pump impeller in curvilinear coordinate system

If circumferential velocity $u=-\omega r<0$ and absolute velocity $c_{3}=-c_{u}<0$, where $\omega$ is the angular velocity and $c_{u}$ is the circumferential component of the absolute velocity, then relative velocity $w_{3}=c_{3}+\omega r=-c_{u}+\omega r>0$ for unit vectors $\vec{e}_{3}^{o}=-\vec{u}^{o}$, also, if circumferential velocity $u=\omega r>0$ and $c_{3}=c_{u}>0$, then $w_{3}=c_{3}-\omega r=c_{u}-\omega r<0$ for $\vec{e}_{3}^{o}=\vec{u}^{o}$.

The suction side of the impeller blade is denoted by " $l$ " and the pressure side is denoted by " $g$ " (Fig. 1), indexes " $a$ " and " $b$ " are: $a=l, b=g$ for $\vec{e}_{3}^{o}=-\vec{u}^{o}$, respectively $a=g, b=l$ for $\vec{e}_{3}^{o}=-\vec{u}^{o}[8]$.

Absolute $(c)$ and relative $(w)$ flow velocities are related, taking into account $\vec{\omega}= \pm \omega \cdot \vec{e}_{z}^{o}$ (in the direction of the rotation axis),

$$
\vec{c}=\vec{w}+\vec{u}=\vec{w}+[\vec{\omega}, \vec{r}], \text { therefore, } \operatorname{rot} \vec{c}=\operatorname{rot} \vec{w}+2 \vec{\omega} .
$$

According to the cosines theorem, the velocity triangle exists: $c^{2}=w^{2}+\omega^{2} r^{2}-2 \omega c_{u}$, therefore, $w^{2}=c^{2}-\omega^{2} r^{2}+2 \omega c_{u}$.

Scalar and vector functions can be considered as an averaged value over the circular coordinate and pulsation component. The averaged pulsation component is equal to zero [5].

\subsection{Averaging of flow parameters in turbo pumps}

Any scalar function $f\left(q_{1}, q_{2}, q_{3}\right)$ can be averaged over circumferential coordinate $q_{3}$ :

$$
\bar{f}\left(q_{1}, q_{2}\right)=\frac{1}{\Delta q_{3}} \int_{q_{3 a}\left(q_{1}, q_{2}\right)}^{q_{33}\left(q_{1}, q_{2}\right)} f\left(q_{1}, q_{2}, q_{3}\right) \mathrm{d} q_{3},
$$

where the circumferential coordinate can be written as $q_{3}=r \varphi$ (Fig. 1). 
Point $\mathrm{M}\left(q_{1}, q_{2}\right)$ in the meridian cross-section of the centrifugal pump impeller belongs to radial coordinate $r=r\left(q_{1}, q_{2}\right)$. All the flow parameters, such as pressure $p$ or vector components (relative $w_{j}$ and absolute $c_{j}$ flow velocity), can be averaged over circumferential coordinate $q_{3}$. The averaging interval is $\Delta q_{3}=q_{3 b}\left(q_{1}, q_{2}\right)-q_{3 b}\left(q_{1}, q_{2}\right)$, where $q_{3 b}\left(q_{1}, q_{2}\right)$ and $q_{3 a}\left(q_{1}, q_{2}\right)$ are equations of two neighboring blade surfaces in the blade passage.

Formula (2) becomes [7, 8]:

$$
\bar{f}\left(q_{1}, q_{2}\right)=\frac{1}{\Delta q_{3}} \int_{q_{3 a}(r)}^{q_{3 b}(r)} f\left(q_{1}, q_{2}, q_{3}(r)\right) \mathrm{d} q_{3}(r)=\frac{1}{\Delta \varphi(r)} \int_{\varphi_{a}(r)}^{\varphi_{b}(r)} f\left(q_{1}, q_{2}, \varphi(r)\right) \mathrm{d} \varphi(r),
$$

where, $\mathrm{d} q_{3}=\mathrm{d} q_{3}(r) / L_{3}$ and $\Delta q_{3}=\Delta q_{3}(r) / L_{3}=r \Delta \varphi(r) / L_{3}$, for $\Delta \varphi(r)=\varphi_{b}(r)-\varphi_{a}(r)$ [deg].

Since coordinate $q_{3}$ is circular, the averaging is obtained over a circular arc in the blade passage area. Depending on the number of numerically calculated flow parameters the integral in Eq. (3) can be resolved numerically using the trapezoidal rule.

In the Ansys CFX software, even only one blade with half of the blade passages on each side can be numerically simulated (in order to reduce the computational time due to impeller symmetry). The same strategy applies to the Fluent software [10]. Anyway, regardless of whether the whole impeller or just a single blade is numerically simulated, only one blade with half of the blade passages around it will be enough for analyzing numerical simulation results, as shown in Fig. 2.

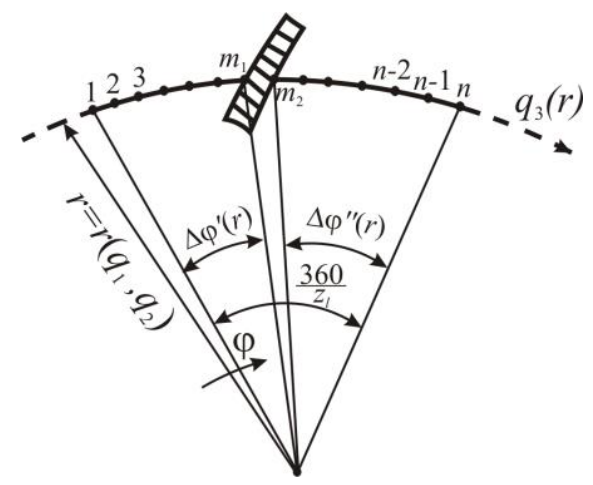

Fig. 2 Averaging over circular coordinate $q_{3}$ (around the blade)

Averaging of the scalar function for the values as given in Fig. 2 can be written:

$$
\bar{f}\left(q_{1}, q_{2}\right)=\frac{1}{\Delta \varphi^{\prime}(r)} \int_{\varphi_{1}(r)}^{\varphi_{m_{1}}(r)} f\left(q_{1}, q_{2}, \varphi(r)\right) \mathrm{d} \varphi(r)+\frac{1}{\Delta \varphi^{\prime \prime}(r)} \int_{\varphi_{m_{2}}(r)}^{\varphi_{n}(r)} f\left(q_{1}, q_{2}, \varphi(r)\right) \mathrm{d} \varphi(r),
$$

where, $\Delta \varphi^{\prime}(r)=\varphi_{m_{1}}(r)-\varphi_{1}(r), \Delta \varphi^{\prime \prime}(r)=\varphi_{n}(r)-\varphi_{m_{2}}(r)[\mathrm{deg}]$

The application of the trapezoidal rule for Eq. (4) yields:

$$
\bar{f}\left(q_{1}, q_{2}\right)=\frac{1}{2 \Delta \varphi^{\prime}(r)}\left(\sum_{j=2}^{m_{1}}\left(f_{j}+f_{j-1}\right)\left(\varphi_{j}-\varphi_{j-1}\right)\right)+\frac{1}{2 \Delta \varphi^{\prime \prime}(r)}\left(\sum_{j=m_{2}+1}^{n}\left(f_{j}+f_{j-1}\right)\left(\varphi_{j}-\varphi_{j-1}\right)\right),
$$


where:

$\Delta \varphi^{\prime}(r)=\varphi_{m_{1}}(r)-\varphi_{1}(r), \Delta \varphi^{\prime \prime}(r)=\varphi_{n}(r)-\varphi_{m_{2}}(r), \varphi[\operatorname{deg}], f_{j}=f_{j}(r), j=1,2, \ldots m_{1}, m_{2}, \ldots, n$.

\subsection{Averaging of derivatives of flow parameters, gradient, divergence and curl}

The corresponding derivatives of the flow parameters can be averaged as follows [7]:

$$
\begin{gathered}
\left(\frac{\overline{\partial f}}{\partial q_{3}}\right)=\frac{1}{\Delta q_{3}} \Delta f, \text { where } \Delta f=f_{b}\left(q_{1}, q_{2}\right)-f_{a}\left(q_{1}, q_{2}\right) \text { and } \\
\left(\overline{\frac{\partial f}{\partial q_{1,2}}}\right)=\frac{1}{\Delta q_{3}} \frac{\partial\left(\Delta q_{3} \bar{f}\right)}{\partial q_{1,2}}-\frac{1}{\Delta q_{3}} \Delta\left(f \frac{\partial q_{3}}{\partial q_{1,2}}\right) \text {, where: } \Delta\left(f \frac{\partial q_{3}}{\partial q_{1,2}}\right)=\left(f \frac{\partial q_{3}}{\partial q_{1,2}}\right)_{b}-\left(f \frac{\partial q_{3}}{\partial q_{1,2}}\right)_{a}
\end{gathered}
$$

Inclination angles of blade profiles, $\beta_{a, b}$ and $\alpha_{a, b}$, are measured in the negative direction of the circumferential flow velocity, as shown in Fig. 3. Also, for a radial pump impeller, the vectors perpendicular to blade surfaces, $\vec{n}_{1 a, b}, \vec{n}_{2 a, b}, \vec{n}_{3 a, b}$, are given in Fig. 3. Thus, the averaged derivative (6) can be transformed into the next equation:

$$
\left(\overline{\frac{\partial f}{\partial q_{1,2}}}\right)=\frac{1}{\Delta q_{3}} \frac{\partial\left(\Delta q_{3} \cdot \bar{f}\right)}{\partial q_{1,2}}+\frac{1}{\Delta q_{3}} \frac{L_{1,2}}{L_{3}} \Delta\left(f \frac{n_{1,2}}{n_{3}}\right) \text {, for } \Delta\left(f \frac{n_{1,2}}{n_{3}}\right)=\left(f \frac{n_{1,2}}{n_{3}}\right)_{b}-\left(f \frac{n_{1,2}}{n_{3}}\right)_{a}
$$

where, $\left(\frac{\partial q_{3}}{\partial q_{1}}\right)_{a, b}=\left(\frac{L_{1}}{L_{3}}\right) \operatorname{ctg} \beta_{a, b}=-\left(\frac{L_{1} n_{1}}{L_{3} n_{3}}\right)_{a, b}$ and $\left(\frac{\partial q_{3}}{\partial q_{2}}\right)_{a, b}=\left(\frac{L_{2}}{L_{3}}\right) \operatorname{ctg} \alpha_{a, b}=-\left(\frac{L_{2} n_{2}}{L_{3} n_{3}}\right)_{a, b}$ for a point $\mathrm{M}\left(q_{1}, q_{2}\right)$.

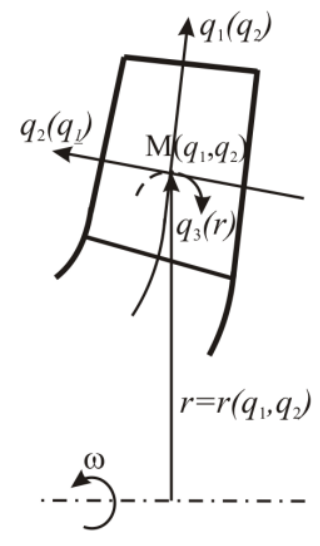

$q_{3}=$ const.

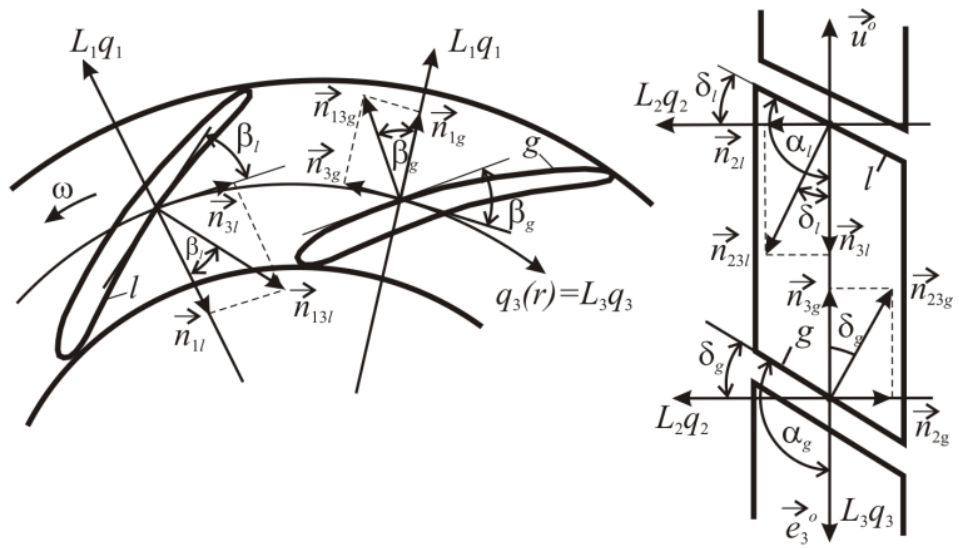

$q_{2}=$ const . $q_{1}=$ const.

Fig. 3 Cross-sections of the centrifugal pump impeller where $\vec{e}_{3}^{o}=-\vec{u}^{o} \quad(a=l, b=g)$ 
Gradient of a scalar function $f$, divergence and curl of any vector $\vec{v}$ become $[7,8]$ :

$$
\begin{aligned}
& \overline{\operatorname{grad} f}=\frac{1}{\Delta q_{3}} \cdot \operatorname{grad}\left(\Delta q_{3} \cdot \bar{f}\right)+\frac{1}{L_{3} \Delta q_{3}} \Delta\left(f \frac{\vec{n}}{n_{3}}\right)=\operatorname{grad} \bar{f}+\frac{1}{L_{3} \Delta q_{3}} \Delta\left(f^{\prime} \frac{\vec{n}}{n_{3}}\right) \\
& \overline{\operatorname{div} \vec{v}}=\frac{1}{\Delta q_{3}} \operatorname{div}\left(\Delta q_{3}, \overline{\vec{v}}\right)+\frac{1}{L_{3} \Delta q_{3}} \Delta\left(\frac{\vec{n}}{n_{3}}, \vec{v}\right)=\operatorname{div} \overline{\vec{v}}+\frac{1}{L_{3} \Delta q_{3}} \Delta\left(\frac{\vec{n}}{n_{3}}, \vec{v}^{\prime}\right) \\
& \overrightarrow{\operatorname{rot} \vec{v}}=\frac{1}{\Delta q_{3}} \operatorname{rot}\left(\Delta q_{3}, \overline{\vec{v}}\right)+\frac{1}{L_{3} \Delta q_{3}} \Delta\left[\frac{\vec{n}}{n_{3}}, \vec{v}\right]=\operatorname{rot} \overline{\vec{v}}+\frac{1}{L_{3} \Delta q_{3}} \Delta\left[\frac{\vec{n}}{n_{3}}, \vec{v}^{\prime}\right]
\end{aligned}
$$

Also, the following relations are obtained:

$$
\overline{\operatorname{grad} f^{\prime}}=\frac{1}{L_{3} \Delta q_{3}} \Delta\left(f^{\prime}, \frac{\vec{n}}{n_{3}}\right), \overline{\operatorname{div} \vec{v}^{\prime}}=\frac{1}{L_{3} \Delta q_{3}} \Delta\left(\frac{\vec{n}}{n_{3}}, \vec{v}^{\prime}\right), \overline{\operatorname{rot} \vec{v}^{\prime}}=\frac{1}{L_{3} \Delta q_{3}} \Delta\left[\frac{\vec{n}}{n_{3}}, \vec{v}^{\prime}\right] .
$$

\subsection{Averaging of fluid flow equations}

There are two equations used for describing a turbulent incompressible fluid flow for the stationary operation of turbo machine, and the first one is continuity equation:

$$
\operatorname{div} \vec{w}=0 .
$$

Neglecting gravity, the flow equation is given by the formulation [13]:

$$
[\vec{w}, \operatorname{rot} \vec{c}]=\operatorname{grad} E,
$$

where $E$ is the energy per unit mass of the fluid of density $\rho$, according to the Bernoulli's integral for relative fluid flow:

$$
E=\frac{p}{\rho}+\frac{w^{2}}{2}-\frac{(r \omega)^{2}}{2}, E=E\left(q_{1}, q_{2}, q_{3}\right) .
$$

Averaging the continuity Eq. (10) over the circular coordinate, it becomes:

$$
\operatorname{div}\left(\Delta q_{3}, \overline{\vec{w}}\right)+\frac{1}{L_{3}} \Delta\left(\frac{\vec{n}}{n_{3}}, \vec{w}\right)=0 .
$$

Equation (13) can be transformed due to the requirement of perpendicularity of vectors $\vec{n}$ and $\vec{w}$, then the second term of Eq. (13) is equal to zero. The remaining part of the equation has been developed and then multiplied with the number of blades $z_{l}=2 \pi / \tau$, where: $\tau=2 \pi / z_{l}$ is an angular blade pitch, $k=\Delta \varphi / \tau=z_{l} \Delta \varphi / 2 \pi$ is the blockage factor, which represents the cross-section reduction due to the real thickness of the impeller blades, and $\Delta q_{3}=r_{o} \cdot \Delta \varphi[7,8]$. Thus, Eq. (13) becomes:

$$
\frac{\partial}{\partial q_{1}}\left(2 \pi r_{o} k L_{3} L_{2} \bar{w}_{1}\right)=-\frac{\partial}{\partial q_{2}}\left(2 \pi r_{o} k L_{3} L_{1} \bar{w}_{2}\right)
$$


Equation (14) is a necessary and sufficient condition for the existence of meridian stream function, $\bar{\psi}_{m}=\bar{\psi}_{m}\left(q_{1}, q_{2}\right)$, where the meridian velocity is $\overline{\vec{w}}_{m}=\bar{w}_{1} \vec{e}_{1}^{o}+\bar{w}_{2} \vec{e}_{2}^{o}$, thus

$$
\frac{\partial \bar{\psi}_{m}}{\partial q_{1}}=-2 \pi r_{o} k L_{3} L_{2} \bar{w}_{2} \text { and } \quad \frac{\partial \bar{\psi}_{m}}{\partial q_{2}}=2 \pi r_{o} k L_{3} L_{1} \bar{w}_{1} .
$$

Equation (15) can be used to calculate the averaged meridian stream function (meridian streamlines) $\bar{\psi}_{m}$, for known averaged velocities and blockage factor. It determines volume flow rate $(Q)$ through axisymmetric cross-sections, $\mathrm{d} \bar{\psi}_{m}=\mathrm{d} Q$

Second term in Eq. (13) can be neglected only in the case of a very thin boundary layer (a fully developed primary turbulent flow with very high Reynolds numbers).

In practice, the fluid flow in turbo pumps is highly turbulent but often showing separations of the boundary layers, when the second term in Eq. (13) should not be neglected and the meridian streamlines should be determined by using the requirement of equal flow rate passing between the axisymmetric flow surface and the hub surface (not using Eq. (15)).

Averaging of the flow Eq. (11), where the flow values can be treated as the sum of mean and fluctuation values, $\vec{w}=\overline{\vec{w}}+\vec{w}^{\prime}$ and $\operatorname{rot} \vec{c}=\overrightarrow{\operatorname{rot} \vec{c}}+(\operatorname{rot} \vec{c})^{\prime}$, it becomes [7, 8]:

$$
[\overline{\vec{w}}, \overline{\operatorname{rot} \vec{c}}]+\overline{\left[\vec{w}^{\prime},(\operatorname{rot} \vec{c})^{\prime}\right]}=\overline{\operatorname{grad} E} .
$$

Equation (16) can be finally written in the next form [8]:

$$
[\overline{\vec{w}}, \operatorname{rot} \overline{\vec{c}}]+\vec{F}^{(1)}+\vec{F}^{(2)}+\vec{F}^{(3)}=\operatorname{grad} \bar{E}
$$

where, $\quad \vec{F}^{(1)}=\left[\overrightarrow{\vec{w}}, \frac{1}{L_{3} \Delta q_{3}} \Delta\left[\frac{\vec{n}}{n_{3}}, \vec{w}^{\prime}\right]\right], \vec{F}^{(2)}=\frac{1}{L_{3} \Delta q_{3}} \Delta\left(E^{\prime}, \frac{\vec{n}}{n_{3}}\right), \vec{F}^{(3)}=\overline{\left[\vec{w}^{\prime}, \operatorname{rot} \vec{w}^{\prime}\right]}$

$$
\text { and } \bar{E}=\frac{\bar{p}}{\rho}+\frac{\bar{w}^{2}}{2}-\frac{(r \omega)^{2}}{2}
$$

Equation (18) represents the forces obtained as the result of averaging, which should be treated as mass forces of the blades acting on the averaged fluid flow. Since $\vec{F}^{(3)}<<\vec{F}^{(1)}$, especially if $\vec{w}$ is changing linearly over circumferential coordinate $q_{3}, \vec{F}^{(3)}$ can be neglected [13]. Also, for axisymmetric flow and model of inviscid fluid, $\vec{F}^{(2)}=0$. But if the fluid flow is not axisymmetric, $\vec{F}^{(2)}$ has to be taken into account. Thus, $\vec{F}=\vec{F}^{(1)}+\vec{F}^{(2)}$ :

$$
\begin{aligned}
& F_{1}^{(1)}=\mp \frac{1}{L_{3} \Delta q_{3}}\left[\Delta\left(\bar{w}_{2} w_{2}^{\prime}+\bar{w}_{3} w_{3}^{\prime}\right) \operatorname{ctg} \beta \pm \bar{w}_{2} \Delta\left(w_{1}^{\prime} \operatorname{ctg} \alpha\right)-\bar{w}_{3} \Delta w_{1}^{\prime}\right] \\
& F_{2}^{(1)}=\mp \frac{1}{L_{3} \Delta q_{3}}\left[\Delta\left(\bar{w}_{3} w_{3}^{\prime}+\bar{w}_{1} w_{1}^{\prime}\right) \operatorname{ctg} \alpha \pm \bar{w}_{1} \Delta\left(w_{2}^{\prime} \operatorname{ctg} \beta\right)-\bar{w}_{3} \Delta w_{2}^{\prime}\right] \\
& F_{3}^{(1)}= \pm \frac{\bar{w}_{1}}{L_{3} \Delta q_{3}}\left[\Delta\left(\bar{w}_{3} \operatorname{ctg} \beta\right) \pm \bar{w}_{2} \Delta\left(w_{3}^{\prime} \operatorname{ctg} \alpha\right)+\bar{w}_{1} \Delta w_{1}^{\prime}+\bar{w}_{2} \Delta w_{2}^{\prime}\right]
\end{aligned}
$$


and $F_{1}^{(2)}= \pm \frac{1}{L_{3} \Delta q_{3}} \Delta\left(E^{\prime}, \operatorname{ctg} \beta\right), \quad F_{2}^{(2)}= \pm \frac{1}{L_{3} \Delta q_{3}} \Delta\left(E^{\prime}, \operatorname{ctg} \alpha\right), \quad F_{3}^{(2)}=-\frac{1}{L_{3} \Delta q_{3}} \Delta E$,

where, $n_{1} / n_{3}=F_{1} / F_{3}=\mp \operatorname{ctg} \beta$ and $n_{2} / n_{3}=F_{2} / F_{3}=\mp \operatorname{ctg} \alpha$.

Assuming axisymmetric flow surfaces (when $\vec{F}^{(2)}=0$ ) the general equation of the averaged axisymmetric flow in centrifugal pump can be obtained. The surfaces are perpendicular to $\vec{F}^{(1)}$ and vector $\vec{n}^{(1)}$ is in the direction of $\vec{F}^{(1)}\left(\left[\vec{n}^{(1)}, \vec{F}^{(1)}\right]=0\right)$, then $\left(\vec{n}^{(1)}, \overline{\vec{w}}\right)=0$. Since $\beta^{(1)}$ and $\alpha^{(1)}$ are corresponding blade angles and considering $\bar{c}_{1}=\bar{w}_{1}$, $\bar{c}_{2}=\bar{w}_{2}, \bar{c}_{3}=\bar{w}_{3}-\omega r$, for $\vec{e}_{3}^{o}=-\vec{u}^{o}$ and $\bar{c}_{3}=\bar{w}_{3}+\omega r$, for $\vec{e}_{3}^{o}=\vec{u}^{o}$, Eq. (11), i.e. Eq. (17) transforms into the general equation of the averaged fluid flow in pump impellers:

$$
\begin{gathered}
\mp c t g \beta^{(1)} \frac{1}{r L_{2}} \frac{\partial\left(r \bar{c}_{3}\right)}{\partial q_{2}} \pm c t g \alpha^{(1)} \frac{1}{r L_{1}} \frac{\partial\left(r \bar{c}_{3}\right)}{\partial q_{1}}+\frac{1}{L_{1} L_{2}}\left(\frac{\partial\left(L_{2} \bar{c}_{2}\right)}{\partial q_{1}}-\frac{\partial\left(L_{1} \bar{c}_{1}\right)}{\partial q_{2}}\right)+\frac{1}{\tilde{c}_{1}} \frac{1}{L_{2}} \frac{\partial \bar{E}}{\partial q_{2}}=0 \\
\text { or, } \mp c \operatorname{ctg} \beta^{(1)} \frac{1}{r L_{2}}\left(\frac{\partial\left(r \bar{w}_{3}\right)}{\partial q_{2}} \mp 2 \omega r \frac{\partial r}{\partial q_{2}}\right) \pm \operatorname{ctg} \alpha^{(1)} \frac{1}{r L_{1}}\left(\frac{\partial\left(r \bar{w}_{3}\right)}{\partial q_{1}} \mp 2 \omega r \frac{\partial r}{\partial q_{1}}\right)+ \\
+\frac{1}{L_{1} L_{2}}\left(\frac{\partial\left(L_{2} \bar{w}_{2}\right)}{\partial q_{1}}-\frac{\partial\left(L_{1} \bar{w}_{1}\right)}{\partial q_{2}}\right)+\frac{1}{\bar{w}_{1}} \frac{1}{L_{2}} \frac{\partial \bar{E}}{\partial q_{2}}=0 .
\end{gathered}
$$

Real and viscous fluids differ from non-viscous ones; and, according to the energy losses, $\bar{E}$, the averaged Bernoulli's integral for relative and absolute flow can be written [8]:

$$
\bar{E}\left(\bar{\psi}_{m}\right)=\bar{E}_{o}\left(\bar{\psi}_{m}\right)-\Delta \bar{E}_{g}\left(\bar{\psi}_{m}\right)=\bar{G}_{o}\left(\bar{\psi}_{m}\right) \pm \omega\left(r \bar{c}_{u}\right)_{o}\left(\bar{\psi}_{m}\right)-\Delta \bar{E}_{g}\left(\bar{\psi}_{m}\right)
$$

where $\bar{E}_{o}\left(\bar{\psi}_{m}\right)$ is averaged mechanical energy of the relative fluid flow in inlet control surface "o", $\Delta E_{g}\left(\bar{\psi}_{m}\right)$ is averaged flow energy loss from the inlet control surface to the arbitrary circular cross-section, when the meridian trace is line $\bar{\psi}_{m}=$ const.

In Eq. 23, $\bar{G}_{o}(\bar{\psi})$ relates to averaged absolute flow, $\bar{G}_{o}(\bar{\psi})=\bar{p}_{o} / \rho+\bar{c}_{o}^{2} / 2$, where $\bar{c}_{u}=-\bar{c}_{3}$ for $\vec{e}_{3}^{o}=-\vec{u}^{o}$ (for “+") and $\bar{c}_{u}=\bar{c}_{3}$ for $\vec{e}_{3}^{o}=\vec{u}^{o}$ (for "-“).

In vaneless parts of the pump, the differential equation of the averaged flow is obtained for $\vec{F}=0$.

\section{NUMERICAL SIMULATION OF FLUID FLOW IN RADIAL PUMP IMPELLER}

The given methodology for obtaining averaged flow surfaces and averaged streamlines in the radial pump impeller is illustrated in the case of a centrifugal pump, which is constructed and tested as the part of the previous project of the Department of Hydroenergetics, Faculty of Mechanical Engineering Niš ("Improving the constructive solution of a centrifugal pump in order to increase its operating area and to improve cavitation characteristics").

It is a centrifugal norm pump (Fig. 4), used mainly for water supply or irrigation purposes, which operates with the following operating parameters: flow rate $Q=30 \mathrm{l} / \mathrm{s}$, pump head $H=14,9 \mathrm{~m}$, number of revolutions $n=1490 \mathrm{~min}^{-1}$, efficiency $\eta=0,78$, inlet 
diameter is $D_{1}=110 \mathrm{~mm}$, outlet diameter $D_{2}=250 \mathrm{~mm}$ (cut to $220 \mathrm{~mm}$ ) and 6 impeller blades. Specific speed is $n_{q}=n \cdot Q^{1 / 2} H^{-3 / 4}=33,3$. Detailed impeller geometry is given in [8].
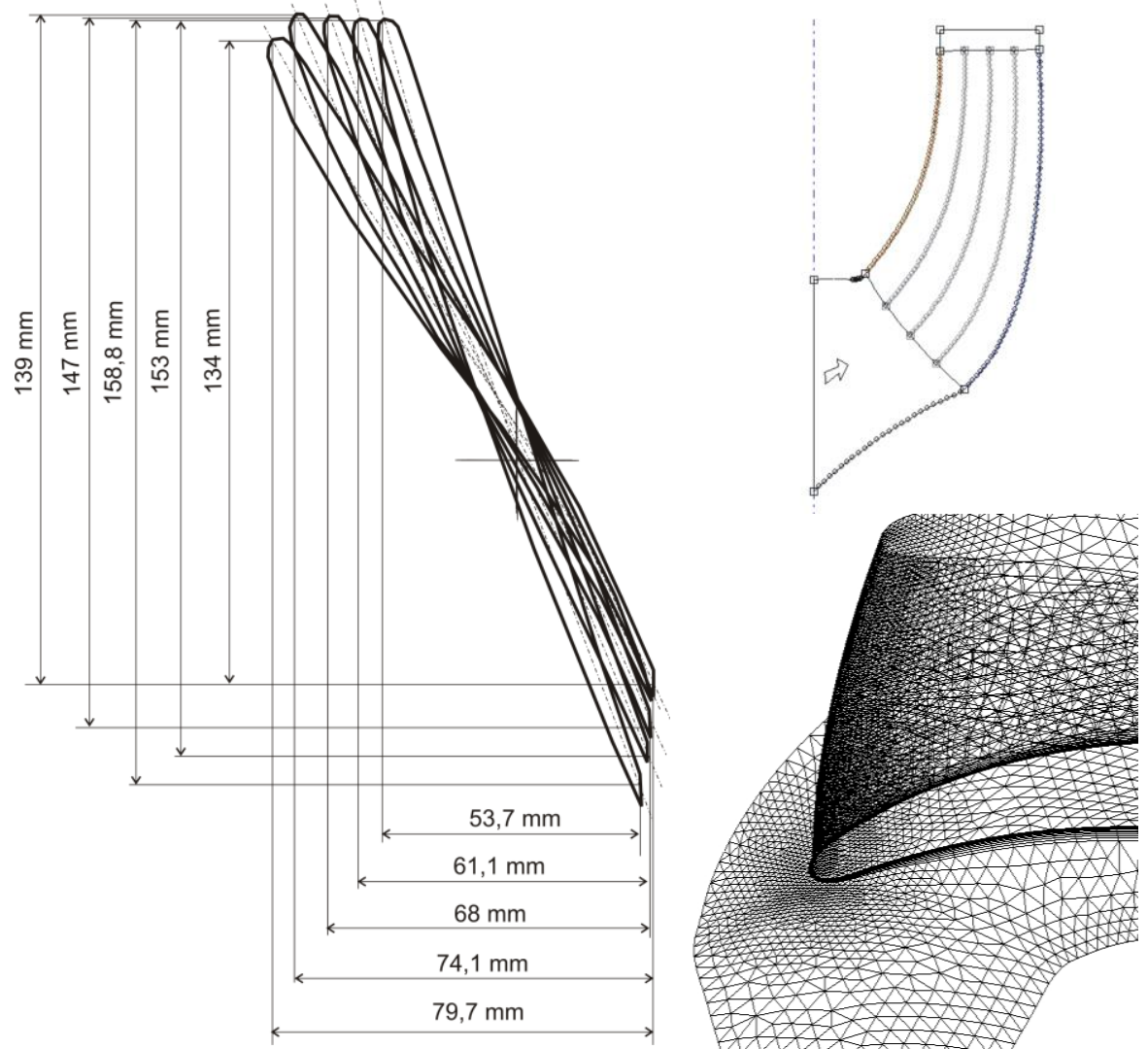

Fig. 4 Geometric model of the centrifugal pump impeller and a detail of the discretization mesh used in CFD

A discretization mesh is generated using the Ansys Workbench BladeGen and it consists of 166998 nodes and 700506 elements, mostly tetrahedral, 589332, pyramidal 463 and wedges 110711, (Fig. 4). Spiral casing is deliberately omitted since in some studies [14] it has been shown that for such models of centrifugal pumps the spiral does not affect too much pump performance characteristics.

Numerical simulations of the centrifugal pump are performed using the Ansys CFX 14.0, which solves RANS equations, with standard $k-\varepsilon$ turbulent model $[15,16]$. Specified conditions are: mass flow at the impeller inlet and static pressure at the outlet. High resolution advection scheme is used. Numerical solving of governing equations continues until the root mean square values of the equation residuals become smaller than $10^{-5}$. 


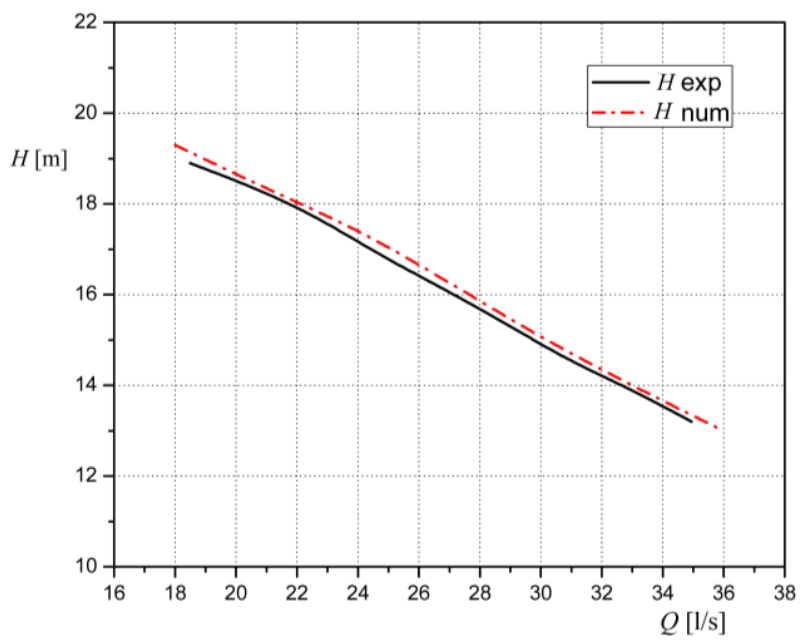

Fig. 5 Numerical and experimental results of the pump head characteristic

The volume flow rate is changed from $18 \mathrm{l} / \mathrm{s}$ to $36 \mathrm{l} / \mathrm{s}$, giving the following operating characteristic chart. Validation of obtained results is presented in Fig. 5; the numerical and experimental curves show a very small difference in the values obtained $(1,7 \%)$.

\section{DETERMinAtion OF MERIDIAN StREAMLINES OF AVERAGED Flow USING THE INTEGRAL CONTINUITY EQUATION}

First of all, it is necessary to determine a series of control cross-sections across meridian lines $\left(L_{k}, k=1,2, \ldots\right)$, as shown in Fig. 6 [8].
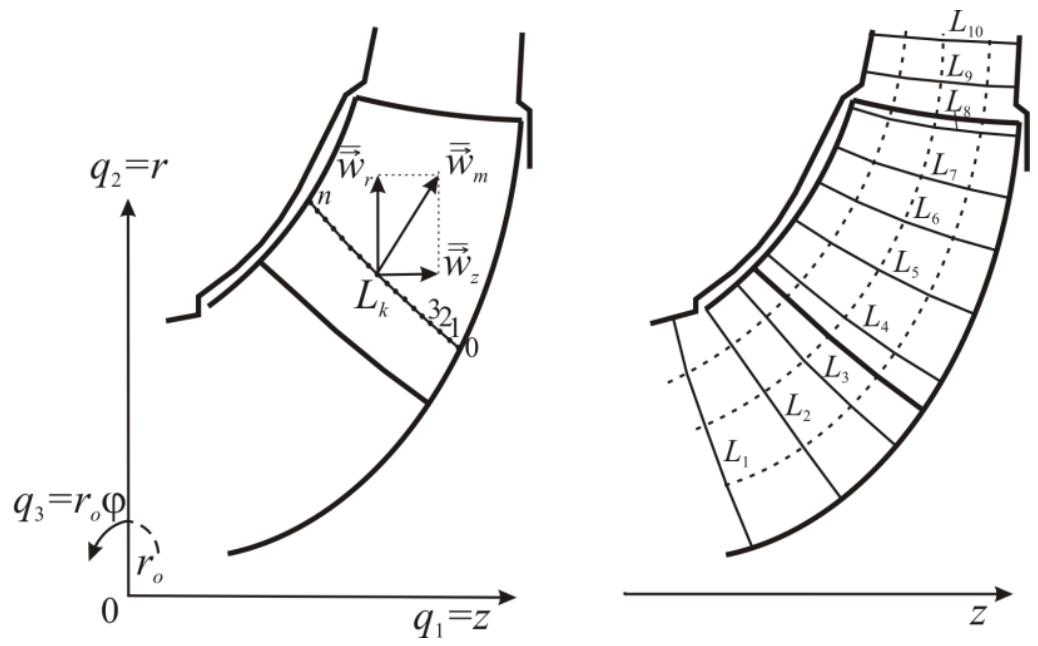

Fig. 6 Control cross-sections 
Each of these lines contains an arbitrary number of calculation points $(n)$ and for every point an averaged flow parameter (such as $w_{r}, w_{z}, w_{\varphi}, c_{r}, c_{z}, c_{\varphi} w^{2}, c^{2}, p$ and $p_{t}$, Eq. (4)) is calculated, using the values obtained by numerical simulations.

Using the integral continuity equation, for each meridian trace represented with a line $l$, the volume flow rate through an axisymmetric flow surface, for $k=z_{l} \Delta \varphi / 2 \pi$ is:

$$
Q_{l}=2 \pi\left[\int_{r_{0}(l)}^{r_{n}(l)} k c_{z} r \mathrm{~d} r-\int_{z_{o}\left(r_{o}\right)}^{z_{n}\left(r_{n}\right)} k c_{r} r \mathrm{~d} z\right] .
$$

In the centrifugal pump impeller, 6 different cross-sections are selected, two of which are outside of the blade area ( $a-a$ in front of the blades and $f-f$ behind the blades) and four cross-sections are in the impeller blade area $(b-b, c-c, d-d$ and $e-e)$, as shown in Fig. 7. These streamlines represent the initial (or the first) approximation. There are nine points on each meridian control line, which are used for averaging all the flow parameters. Along every circular arc passing through these points, there are ten evenly distributed points in which all the flow parameters are obtained by the CFD.

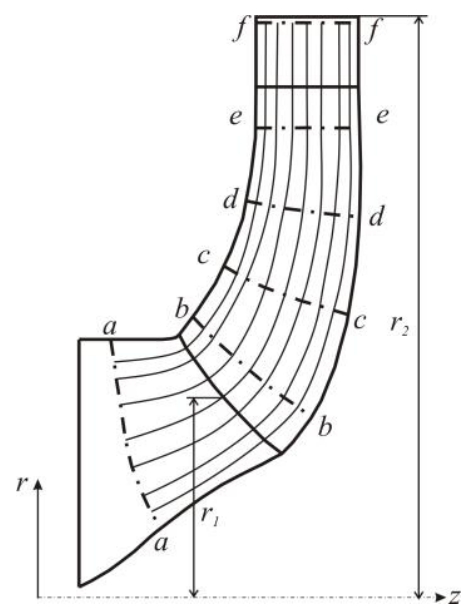

Fig. 7 Designed meridian cross-sections

The following values are needed: the blockage factor due to blade thickness, $k=z_{l} \Delta \varphi / 2 \pi$ number of blades $z_{l}=6$, and angle $\Delta \varphi \leq 60^{\circ}$. Note that in blade area $k<1$, and in vaneless space $k=1$.

Volume flow rates in $j$ cross-section of the pump impeller can be calculated:

$$
Q_{j}=Q_{j-1}+\pi\left(f_{j}+f_{j-1}\right)\left(r_{j}-r_{j-1}\right)+\pi\left(-\left(F_{j}+F_{j-1}\right)\left(z_{j}-z_{j-1}\right)\right), \text { for } j=1,2, \ldots, n
$$

where, $Q_{\mathrm{o}}=0$, and $f$ and $F$ are functions calculated knowing the values of averaged axial $\left(\bar{w}_{z}\right)$ and radial relative velocity $\left(\bar{w}_{r}\right)$ in the pump impeller:

$$
\begin{aligned}
& f_{j}=\left(k r \bar{w}_{z}\right)_{L_{k . j}}=f\left(z_{j}, r_{j}\right)=k\left(z_{j}, r_{j}\right) \cdot r\left(z_{j}, r_{j}\right) \cdot \bar{w}_{z}\left(z_{j}, r_{j}\right), \text { for } j=1,2, \ldots, n \\
& F_{j}=\left(k r \bar{w}_{r}\right)_{L_{k . j}}=F\left(z_{j}, r_{j}\right)=k\left(z_{j}, r_{j}\right) \cdot r\left(z_{j}, r_{j}\right) \cdot \bar{w}_{r}\left(z_{j}, r_{j}\right), \text { for } j=1,2, \ldots, n
\end{aligned}
$$


Flow parameters are obtained by numerical simulation of the flow in the pump impeller, for the nominal operating pump regime. The volume flow rates are calculated for all cross-sections $(a, b, c, d, e, f)$ and the average value is $Q=0,0282 \mathrm{~m}^{3} / \mathrm{s}=28,21 / \mathrm{s}$, obtaining an error of $6 \%$ mostly due to blade thickness and flow separation from the blade surfaces and reverse fluid flow. In cross-sections $a$ - $a$ and $f-f$, which are outside the blade area (in front and behind the impeller blades), the volume flow rates are close to the designed nominal value. Thus, the values obtained are 29,436 1/s and 29,945 1/s, respectively.

\subsection{Correction of meridian streamlines in radial pump impeller}

Considering that in the observed control cross-sections the flow rates between the hub and these surfaces are not equal, it is clear that the assumed axisymmetric flow surfaces in the initial approximation are not averaged meridian streamlines. Therefore, the next step is the correction of streamlines. Fig. 8 shows the initial meridian line $(j)$, the corrected meridian line $\left(j^{\prime}\right)$, and the distances between these lines and the next (or neighboring) line, where $k-k$ is the flow cross-section perpendicular to the meridian streamlines.

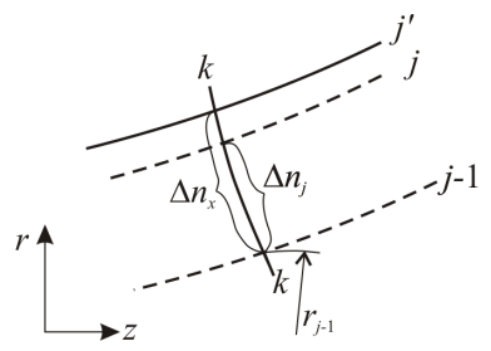

$$
\begin{gathered}
\Delta n_{j}=\sqrt{\Delta z_{j}^{2}+\Delta r_{j}^{2}} \\
\Delta z_{j}=z_{j}-\mathrm{Z}_{j-1}, \Delta r_{j}=r_{j}-r_{j-1} \\
\Delta n_{x}=\sqrt{\Delta z_{x}^{2}+\Delta r_{x}^{2}} \\
\Delta z_{x}=z_{x}-\mathrm{Z}_{j-1}, \Delta r_{x}=r_{x}-r_{j-1}
\end{gathered}
$$

Fig. 8 An illustration for the correction methodology

Volume flow rates between the streamlines can be calculated using the mean values of meridian velocity $\left(c_{\text {m.avg }}^{(j)}\right)$ :

$$
\begin{aligned}
& \Delta Q_{j}=2 \pi r_{j-1} \Delta n_{j} c_{m . a v g}^{(j)}, \text { for } c_{m . a v g}^{(j)}=\frac{1}{2}\left(c_{m . j-1}+c_{m . j}\right) \\
& \Delta Q_{x}=2 \pi r_{j-1} \Delta n_{x} c_{m . a v g}^{\left(j^{\prime}\right)}, \text { for } c_{m . a v g}^{\left(j^{\prime}\right)}=\frac{1}{2}\left(c_{m . j-1}+c_{m . x}\right)
\end{aligned}
$$

Assuming a linear change of the meridian velocity along meridian traces $\Delta n_{j}$ and $\Delta n_{x}$, the relation can be obtained:

$$
\frac{\Delta Q_{x}}{\Delta Q_{j}}=\frac{2\left(\Delta n_{x} / \Delta n_{j}\right)+\mathrm{A}\left(\Delta n_{x} / \Delta n_{j}\right)^{2}}{(2+\mathrm{A})}, \quad \text { where } \mathrm{A}=\frac{c_{m . j}}{c_{m . j-1}}-1
$$

Since

$$
Q_{x}-Q_{j}=\Delta Q_{x}-\Delta Q_{j}, \text { then } \frac{\Delta Q_{x}}{\Delta Q_{j}}=1+\frac{Q_{x}-Q_{j}}{\Delta Q_{j}} .
$$

and the quadratic equation is obtained:

$$
\mathrm{A}\left(\frac{\Delta n_{x}}{\Delta n_{j}}\right)^{2}+2\left(\frac{\Delta n_{x}}{\Delta n_{j}}\right)-\mathrm{B}=0, \text { for } \mathrm{B}=(2+\mathrm{A})\left(1+\frac{Q_{x}-Q_{j}}{\Delta Q_{j}}\right) .
$$


There are two possible solutions of Eq. (30):

$$
\begin{gathered}
\text { if } \mathrm{A} \neq 0, c_{m . j} \neq c_{m . j-1} \text {, then } \frac{\Delta n_{x}}{\Delta n_{j}}=\frac{1}{\mathrm{~A}}(-1 \pm \sqrt{1+\mathrm{AB}}) \quad \text { and } \\
\text { if } \mathrm{A}=0, c_{m \cdot j}=c_{m \cdot j-1} \text {, then } \frac{\Delta n_{x}}{\Delta n_{j}}=1+\frac{Q_{x}-Q_{j}}{\Delta Q_{j}} .
\end{gathered}
$$

Note that as a result of Eq. (31) one should take only a real and positive solution.

In Fig. 9 the initial approximation of the meridian streamlines (dashed lines) and the corrected meridian streamlines (full lines) are presented. A noticeable difference is clearly visible but in some parts of the impeller there is a greater difference of the corrected streamlines, compared to the initially assumed.

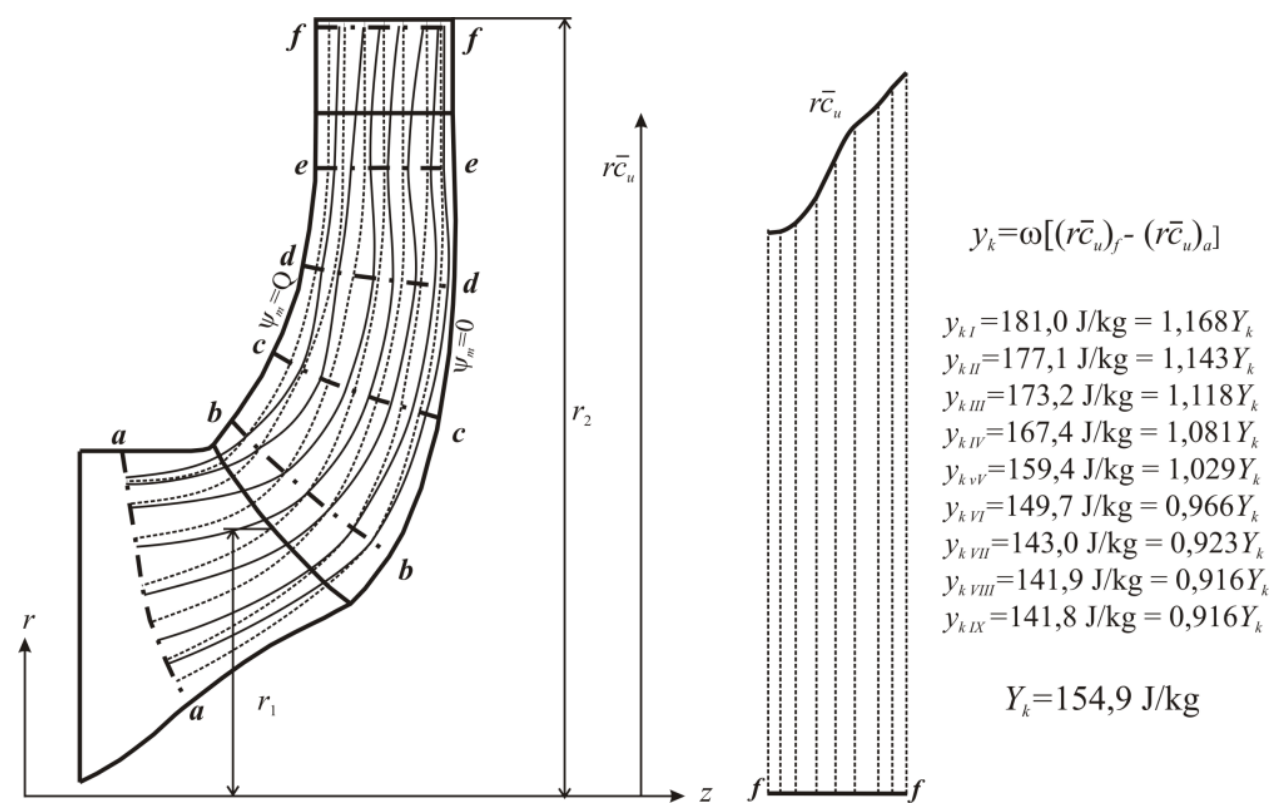

Fig. 9 Meridian streamlines of averaged flow and $r \bar{c}_{u}$ distribution in the impeller outlet

\subsection{Calculation of specific work of pump elementary stages and torque}

The specific work of elementary stages in the pump impeller can be calculated:

$$
y_{k}\left(S_{j}\right)=\omega\left[\left(r \bar{c}_{u}\right)_{f}-\left(r \bar{c}_{u}\right)_{a}\right]
$$

In Fig. 9 a change of the specific work in an outlet cross-section $(f-f)$, calculated for the given example of centrifugal pump is presented. It clearly shows an unequal distribution of $r \bar{c}_{u}$ from the hub to the shroud of the pump impeller. First three cross-sections have similar values of specific work ( $\approx 15 \%$ smaller than design value). In the next (fourth) cross-section 
this value increases, obtaining the design value in the next 3 cross-sections. In the last two cross-sections the specific work exceeds design value up to even $35 \%$.

The common practice in the design of all types of turbomachinery is the assumption of equality of the specific work of elementary stages.

There are some earlier suggestions proposing the use of an unequal distribution of the specific work of elementary stages in turbomachinery designing $[17,18]$. The justification of such an assumption (unequal distribution of $r \bar{c}_{u}$ ) is shown in Fig. 9.

The pump impeller torque can be calculated using the formula:

$$
M_{k}^{(P)}=M_{k}(1,2)=\int_{A_{2}} \operatorname{\rho r} \omega \bar{c}_{u}\left(\overline{\vec{c}}_{m}, \mathrm{~d} \vec{A}\right)-\int_{A_{1}} \rho r \omega \bar{c}_{u}\left(\overline{\vec{c}}_{m}, \mathrm{~d} \vec{A}\right)=M_{k}(2)-M_{k}(1),
$$

where $A_{1}$ and $A_{2}$ are control cross-sections on inlet (1) and outlet (2) of the pump impeller. Therefore,

$$
M_{k}(1,2)=2 \pi \rho \int_{L_{1,2}} r^{2} \bar{c}_{u} \bar{c}_{z} d r-2 \pi \rho \int_{L_{1,2}} r^{2} \bar{c}_{u} \bar{c}_{r} d z,\left(L_{1,2}=L_{1,2}(z, r)\right)
$$

If the calculation points are uniformly and densely distributed along control lines $L_{1}$ and $L_{2}\left(n_{1}, n_{2} \geq 10\right)$, then the integrals of Eq. (34) can be determined, with good accuracy, using the trapesoidal rule. Using the notation, for $j=0,1,2, \ldots \mathrm{n}_{1,2}$ :

$$
\begin{gathered}
z_{j}^{(1,2)}=z_{1,2 . j}, \quad r_{j}^{(1,2)}=r_{1,2 . j}, \\
g_{j}^{(1,2)}=r^{2} \bar{c}_{u} \bar{c}_{z}=g_{j}^{(1,2)}\left(z_{j}^{(1,2)}, r_{j}^{(1,2)}\right), \quad G_{j}^{(1,2)}=r^{2} \bar{c}_{u} \bar{c}_{r}=G_{j}^{(1,2)}\left(z_{j}^{(1,2)}, r_{j}^{(1,2)}\right)
\end{gathered}
$$

Equation (34), i.e. Eq. (35), for inlet and outlet cross-sections, becomes:

$$
M_{k}(1,2)=\pi \rho \sum_{j=1}^{n_{1,2}}\left(g_{j}^{(1,2)}+g_{j-1}^{(1,2)}\right)\left(r_{j}^{(1,2)}-r_{j-1}^{(1,2)}\right)-\pi \rho \sum_{j=1}^{n_{1,2}}\left(G_{j}^{(1,2)}+G_{j-1}^{(1,2)}\right)\left(z_{j}^{(1,2)}-z_{j-1}^{(1,2)}\right)
$$

In the given example of a centrifugal pump, it is obtained $M_{k}=29,785 \mathrm{Nm}$, which differs for only $5 \%$ from the value obtained by numerical simulation.

\section{CONCLUSION}

The principle and method of averaging flow parameters and flow equations, using the results of numerical simulations of flow in the centrifugal pump impeller, are presented in the paper. Meridian streamlines of the averaged flow are obtained, as well as the distribution of specific work in the impeller. The specific work distribution in the impeller outlet shows unequal distribution, which indicates it deviates more or less from the pre-designed values. The impeller torque was estimated as well. Such information is of the greatest importance in the design and calculation of the pump impeller. The meridian streamlines in the centrifugal pump impeller should be corrected during the design process. Using the numerical simulation data, according to the procedure described in the paper, much more relevant information is available to the pump designer. At the same time, this procedure shortens the time required for pump model developing and testing. 
Acknowledgements: The paper is a part of the research done within the project TR33040, "Revitalization of existing and designing new micro and mini hydropower plants (from $100 \mathrm{~kW}$ to $1000 \mathrm{~kW}$ ) in the territory of South and Southeast Serbia", supported by the Ministry of Science and1 Technological Development of the Republic of Serbia.

\section{REFERENCES}

1. Obradović, N., 1973, Basics of Turbomachinery, Gradjevinska knjiga, Belgrade, Serbia (in Serbian).

2. Babić, M., Stojković, S., 1990, Basics of Turbomachinery: operating principles and mathematical modeling, Naučna knjiga, Belgrade, Serbia (in Serbian).

3. Ristić, B., 1987, Pumps and Fans, Naučna knjiga, Belgrade, Serbia (in Serbian).

4. Krsmanović, Lj., Gajić, A., 2005, Turbomachinery - theoretical basics, University of Belgrade, Faculty of Mechanical Engineering Belgrade, Belgrade, Serbia (in Serbian).

5. Voronjec, K., Obradović, N., 1973, Fluid Mechanic, Gradjevinska knjiga, Belgrade, Serbia (in Serbian)

6. Lakshminarayana, B., 2002, Fluid Dynamics and Heat Transfer of Turbomachinery, John Wiley \& Sons, Inc., New York, USA.

7. Bogdanović-Jovanović, J.B, Bogdanović, B.P, Milenković, D.R., 2012, Determination of Averaged axisymmetric flow surfaces according to results obtained by numerical simulation of flow in turbomachinery, Thermal Science, 16 (Suppl.2), pp. 647-662.

8. Bogdanović-Jovanović, J., 2014, Determination of Averaged Axisymmetric flow in hydraulic turbomachinery runner, PhD Thesis, University of Niš, Faculty of Mechanical Engineering, Serbia, 263p

9. Yedidiah, S., 2008, A Study in The Use of CFD In The Design of Centrifugal Pumps, Engineering Applications of Computational Fluid Mechanics, 2(3), pp. 331-343.

10. Stuparu, A., Resiga, R., Muntean, S, 2011, A New Approach in Numerical Assessment of the Cavitation Behaviour of Centrifugal Pumps, International Journal of Fluid Machinery and Systems, 4(1), pp. 104-113.

11. Tan, M.G., He, X.H., Liu, H.L., Dong, L., Wu, X.F., 2016, Design and analysis of a radial diffuser in a single stage centrifugal pump, Engineering Application of Computational Fluid Mechanics, 10(1), pp. 500-511.

12. An, Z., Zhounian, L., Peng, W., Linlin, C., Dazhuan, W., 2015, Multi-objective optimization of a low specific speed centrifugal pump using an evolutionary algorithm, Engineering Optimization, 48(7), pp. 1251-1274.

13. Etinberg, I.E., Rauhman, B.S., 1978, Hydrodynamics of hydraulic turbines, Sent Petersburg, Russia.

14. Stamenković, Ž., Bogdanović-Jovanović, J., Manojlović, J., 2013, Determination of centrifugal pump operating parameters in turbine operating regime, Proceedings, 16. Symposium on Thermal Science and Engineering of Serbia, Sokobanja, Serbia, pp. 846-855.

15. Ferziger, J.H., Peric, M., 2002, Computational methods for fluid dynamics, Springer, Berlin, Heidelberg.

16. Casey, M., 2004, Best practice advice for turbomachinery internal flows, QNET-CFD Network Newsletter (Thematic Network For Quality and Trust in the Industrial Application of CFD), 2(4), pp. 40-46.

17. Bogdanović-Jovanović, J., Bogdanović, B., Božić, I., 2014, Design of small bulb turbines with unequal specific work distribution of the reunner's elementary stages, Facta Universitatis, series: Mechanical Engineering, 12(1), pp. 73-84.

18. Bogdanović, B., Spasić, Ž., Bogdanović-Jovanović, J., 2012, Low-Pressure Reversible Axial Fan Designed with Different Specific Work of Elementary Stages, Thermal Science, 16(Suppl.2), pp. S605-S615. 